\title{
Determining the zero-point calibration for AGN black hole mass estimates
}

\author{
Christopher A. Onken ${ }^{1}$, Laura Ferrarese ${ }^{2}$, David Merritt ${ }^{2,3}$, \\ Bradley M. Peterson ${ }^{1}$, Richard W. Pogge ${ }^{1}$, \\ Marianne Vestergaard ${ }^{1,4}$ and Amri Wandel ${ }^{5}$ \\ ${ }^{1}$ Department of Astronomy, The Ohio State University, 140 West 18th Avenue, Columbus, \\ OH 43210; onken, peterson, pogge@astronomy.ohio-state.edu \\ ${ }^{2}$ Department of Physics and Astronomy, Rutgers University, 136 Frelinghuysen Road, \\ Piscataway, NJ 08854; lff@physics.rutgers.edu \\ ${ }^{3}$ Current address: Department of Physics, Rochester Institute of Technology, 84 Lomb \\ Memorial Drive, Rochester, NY 14623; drmsps@rit.edu \\ ${ }^{4}$ Current address: Steward Observatory, University of Arizona, 933 North Cherry Avenue, \\ Tucson, AZ 85721; mvestergaard@as.arizona.edu \\ ${ }^{5}$ Racah Institute, Hebrew University, Jerusalem 91904, Israel; amri@frodo.fiz.huji.ac.il
}

\begin{abstract}
By fitting to the quiescent galaxy $M_{\mathrm{BH}}-\sigma_{*}$ relation, we calculate the average shift required to scale reverberation-mapped AGN masses to the same zero-point. We use reanalyzed virial products $\left(r V^{2} / G\right)$ and both new and published velocity dispersions to find the offset in the AGN calibration. This scaling factor, $\langle f\rangle$, accounts for the detailed dynamics and geometry of the broad-line region (BLR). Finally, we confirm the rough correlation between $\sigma_{*}$ and FWHM([O III]) for these 16 AGNs.
\end{abstract}

We use the strong correlation linking a galaxy's black hole mass, $M_{\mathrm{BH}}$, to its stellar velocity dispersion, $\sigma_{*}$, to calibrate the $M_{\mathrm{BH}}$ values found for AGNs via reverberation mapping (RM). RM analysis yields the virial product, $M_{r e v}=r V^{2} / G$, where $r$ is measured by the time delay of the emission lines in response to changes in the continuum, and $V$ is measured from the dispersion of the rms line profile. $M_{r e v}$ is related to the black hole mass as $M_{\mathrm{BH}}=f M_{r e v}$, where $f$ accounts for the kinematics and shape of the BLR.

Simple models for the value of $f$ were assumed in earlier work, which found that AGNs are broadly consistent with the quiescent galaxy $M_{\mathrm{BH}}-\sigma_{*}$ relationship. Our approach relies on the assumption that the two relationships are, in fact, identical; the slope of the AGN relation was then fixed to that of the inactive galaxies, and the normalization offset determined the ensemble average scale factor, $\langle f\rangle$. The slope of the $M_{\mathrm{BH}}-\sigma_{*}$ relation is still under debate, so we chose the two most prominent values near the ends of the quoted range: 4.58 (Ferrarese 2002; F02) and 4.02 (Tremaine et al. 2002; T02).

With new measurements of $\sigma_{*}$ for six AGNs and additional values from the literature, we used a sample of 16 AGNs with $M_{\text {rev }}$ data (from Peterson et al. 2004) to determine $\langle f\rangle$. With the slope fixed to the F02 (T02) value, we find $\langle f\rangle=5.5 \pm 1.9(5.5 \pm 1.6)$. The data are plotted in Figure 1, which also shows the $M_{\mathrm{BH}}$ scale on the right-hand axis, obtained by multiplying by $\langle f\rangle$.

Other investigators have used $\operatorname{FWHM}([\mathrm{O} \mathrm{III}])$ as a proxy for $\sigma_{*}$. With [O III $]$ measurements tabulated by Nelson (2000), we examined the correlation between $\sigma_{*}$ and FWHM([O III] ) for our 16 AGNs. Significant scatter was found for individual objects, but the two measures are in approximate agreement. 


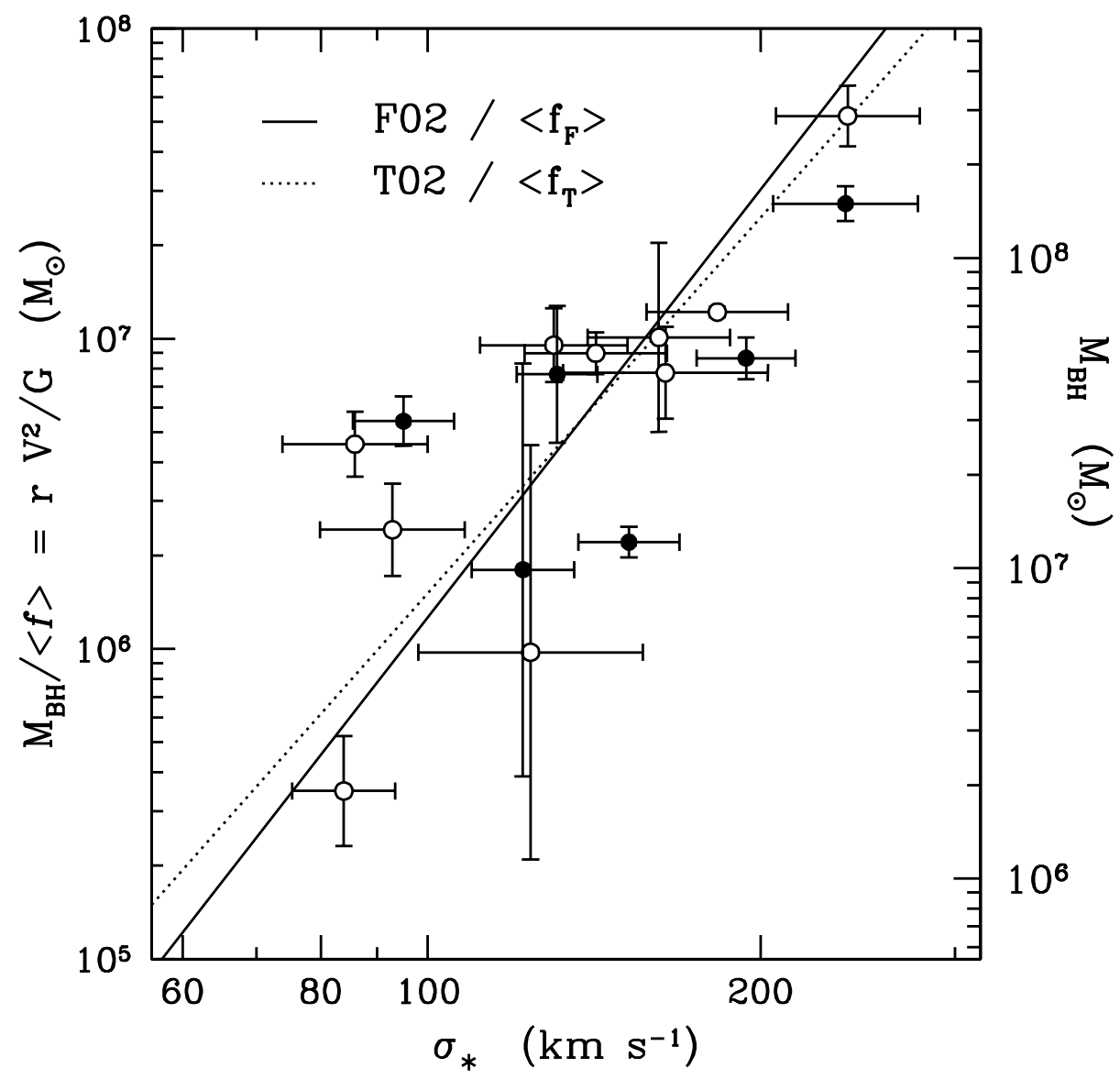

Figure 1. Filled points indicate new $\sigma_{*}$ measurements; open points are published values. $M_{\mathrm{BH}} / f$ data is from Peterson et al. (2004). Solid (dotted) line indicates F02 (T02) slope, with normalization scaled down by $\langle f\rangle$.

\section{References}

Ferrarese, L. 2002, in Current High-Energy Emission Around Black Holes, ed. C.-H. Lee \& H.-Y. Chang (Singapore: World Scientific), 3 (F02; astro-ph/0203047)

Nelson, C. H. 2000, ApJ, 544, L91

Peterson, B. M., et al. 2004, ApJ, submitted

Tremaine, S., et al. 2002, ApJ, 574, 740 (T02) 